\title{
Thermal State Visualization of Machining Workpiece by Means of a Sensor-Configured Heat Conduction Simulation*
}

\begin{abstract}
Koji TERAMOTO**, Ryuichi TANAKA***, Tohru ISHIDA*** and Yoshimi TAKEUCHI ${ }^{\dagger}$
This paper deals with an adaptive visualization method of the thermal state in machining operation. The visualization is expected to enhance the recognition ability of operators. First, a framework of thermal state visualization is proposed. Then, a sensor-configured heat conduction simulation is proposed as an estimation method of thermal states of the machining workpiece. The method is based on the association of sensed data with the process simulation. As an implementation of the proposed estimation method, a prototype system is developed. After illustrating the configuration of the prototype system, results of a simple example are shown. The results indicate the effectiveness of the adaptive estimation. The influence of measurement points are also evaluated based on the case study. Finally, conclusions and future works are presented.
\end{abstract}

Key Words: Operation Support, Thermal State, Visualization, Endmilling

\section{Introduction}

Recently, most manufacturers have been required to improve their manufacturing process. The features of recent requirements can be summarized as small-batch, hiquality and agile production. Endmilling is one of the most effective fabrication processes to meet the requirements. Concerning the milling operation, the thermal behavior of a machining workpiece has become an important aspect because dry machining and MQL machining have become the standard approach to environmentally conscious machining.

On the other hand, as shown in ISO12100 activities ${ }^{(1)}$, the safety of operators has become an important issue. This safety requirement usually results in the separation of

* Received 28th October, 2005 (No. 05-4222)

** Department of Mechanical Engineering, Graduate School of Engineering, Osaka University, 2-1 Yamadaoka, Suita, Osaka 565-0871, Japan.

E-mail: teramoto@mech.eng.osaka-u.ac.jp

*** Department of Mechanical Engineering, Graduate School of Engineering, Osaka University.

E-mail: rtanaka@cape.mech.eng.osaka-u.ac.jp

**** Department of Mechanical Engineering, Graduate School of Engineering, Osaka University.

E-mail: ishidat@mech.eng.osaka-u.ac.jp

$\doteqdot$ Department of Mechanical Engineering, Graduate School of Engineering, Osaka University.

E-mail: takeuchi@mech.eng.osaka-u.ac.jp the machining process from human operators. This trend will result in a decrease of operators' ability to supervise the machining process, because the observation is the most fundamental activity in understanding the machining process. So, enhancing the recognition ability of operators is required as a method to avoid this decrease in operators' ability.

It is well known that visual information plays an effective role in human cognition. Furthermore, Augmented Reality (AR) ${ }^{(2),(3)}$ technology has become widely applicable for various diagnostic applications and provides an effective presentation method of visual information. Based on the circumstances mentioned above, on-site visualization of workpiece states is considered as a supporting method for operators' recognition ability.

Figure 1 illustrates a schematic diagram of on-site visualization of machining states. The visualization system consists of the simulator, sensors, the state estimator, data repository and a human interface. By using this system, operators perceive three information: direct perception, the current machining state and expected machining states. In spite of the separation of the machining process and operators for safety reasons, a certain amount of information, such as the sounds of machining or color of chip, can be observed. Furthermore, operators can see the visualized information in the actual machining ambience. Then, operators are expected to evaluate the machining state during the machining process by combining visualized informa- 


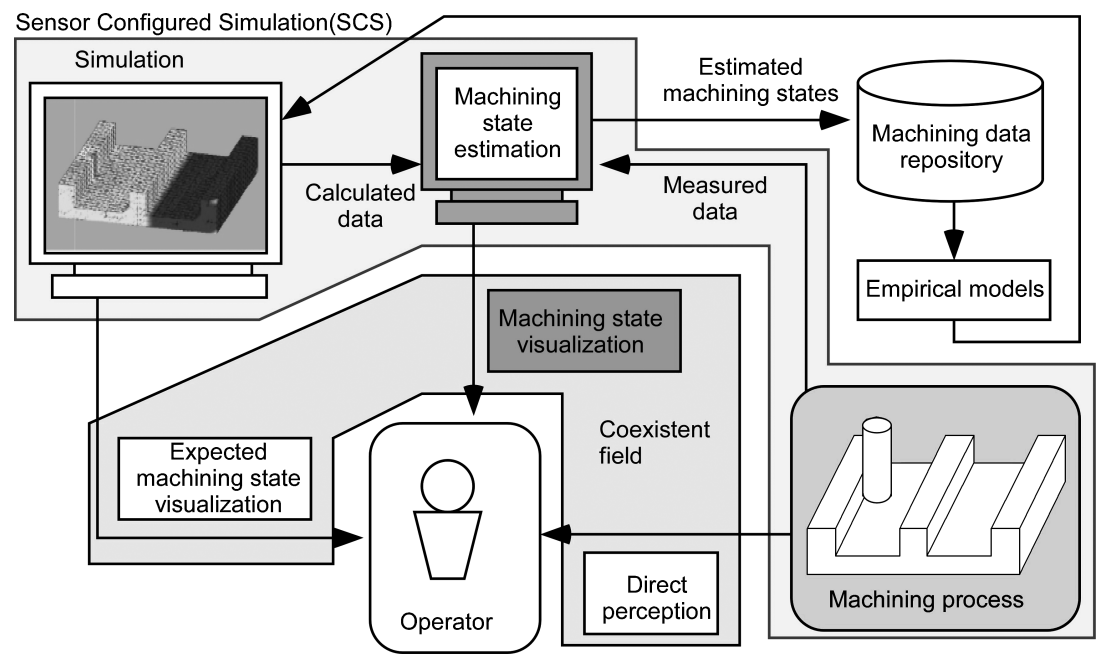

Fig. 1 Framework of on-site visualization of machining states

tion and their own perceptions. Moreover, they can also refer to the expected machining state based on the preprocess simulation $^{(4)}$. On-site visualization is expected to have the following four advantages.

- By visualizing various types of information such as workpiece temperature, operators can recognize the machining process from various aspects.

- The coexistence of the real phenomenon and visualized information helps operators to assess their own sensations and indicated information. This is expected to reinforce their experience.

- By comparing the estimated workpiece states with the pre-process simulation results, it is easier to diagnose the machining process.

- Based on the results of on-site estimation, empirical process models can be generated by applying data mining technology.

In order to provide appropriate support, an accurate state estimation is an essential technological problem to be addressed. In this paper, accurate state estimation of the workpiece temperature under variable situations is discussed to realize reliable on-site visualization. A Sensor Configured Simulation (SCS) has been proposed as an estimation method of the workpiece state ${ }^{(5)}$. The basic concept of the SCS method is to combine local measured data and a machining simulation.

\section{Framework of Sensor-Configured Heat Conduc- tive Simulation}

There have been many researches to estimate the temperature distribution of workpiece ${ }^{(6),(7)}$. Most of the researches have been based on the Finite Element Method (FEM) or the Finite Differential Method (FDM). In order to simulate the machining process as a thermal conduction process, it is necessary to prepare the thermal properties in advance to evaluate the workpiece temperature. Required thermal properties are a heat flux from the cutting tool and heat transfer coefficients between the workpiece surface and the environment and so on.

In spite of the former research, the thermal behavior of a workpiece still cannot be estimated accurately. One reason for this is that there are many uncertainties in the machining operation. The uncertainties come from the variations in the machining situation, such as air blast or swarf accumulation. In order to adapt to these variations, a further mechanism, which helps to compensate for the influence of variations, is required. As mentioned in the first section, we have proposed a machining state estimation framework by associating measured data and analysis results. The developed estimation method is based on the proposed framework. A parameter-tuning procedure is integrated to the process simulation. In order to develop the proposed estimation, an adaptive thermal state estimation procedure should have three functions. They are data measurement, simulation and state estimation. The mechanism for the parameter tuning plays essential roles in state estimation. Furthermore, synchronization of actual measured temperature and simulated situation is an important problem to develop an actual system. Figure 2 illustrates a framework of the SCS estimation method. The proposed framework connects conventional simulation procedures to real phenomena. This connection is expected to enhance the quality of state estimation.

From the point of view of problem solving, the SCS method is understood as an inverse problem of the FEMbased simulation. Based on the standard FEM formulation, the field of the workpiece temperature is described in the set of certain nodal temperatures $\{T\}$. Let us denote a heat conductivity matrix, a heat capacity matrix and a nodal vector by $[K],[C]$ and $\{F\}$ respectively. The differential equation which represents the state is expressed as follows:

$$
\left(\frac{[C]}{\Delta t}+[K]\right)\{T\}=\frac{[C]}{\Delta t}\left\{T^{-}\right\}+\{F\}
$$




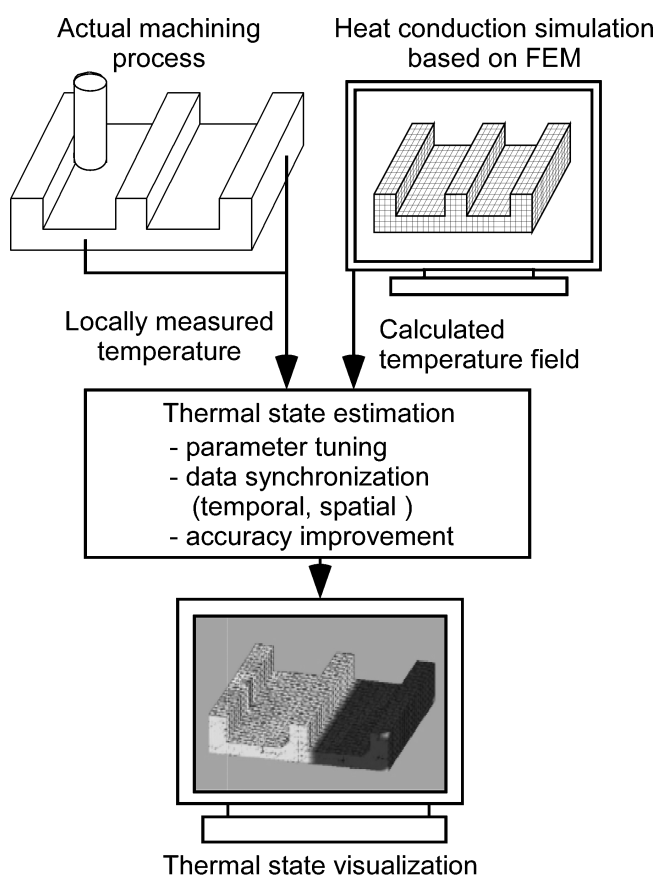

Fig. 2 Framework of sensor-configured heat conduction simulation

where $\left\{T^{-}\right\}$indicates the nodal temperatures in previous time step. On the basis of this representation, the state estimation in the SCS method can be formulated as a determination of $[K]$ and $\{F\}$, which combines the Eq. (1) and measured data.

From the research into the ill-posedness of the inverse problems ${ }^{(8)}$, the total degree of freedom of parameters which should be determined must be smaller than the amount of measured information. In this estimation, former FEM researches show that the number of estimation parameters is considered rather small. Concretely, heat flux and heat transfer coefficients are the parameters which should be estimated. This indicates that the point measurements method like thermocouples is applicable to the proposed estimation.

\section{Estimation Procedure of Sensor-Configured Heat Conduction Simulation}

Figure 3 illustrates the process flow of the prototype system developed for the thermal state estimation. The prototype system consists of two major modules. These are a data collector and a state estimator. The system acquires temperatures of predetermined points by using thermocouples when a data acquisition request message is sent to the data collector. The minimum interval of data collection is one second. A state estimator is developed based on an optimization method which searches parameters. The Quasi-Newton method is employed as an optimization method. FEM analysis is provided to evaluate the optimization candidates. After iterating the optimization procedures, adequate parameters are selected so as

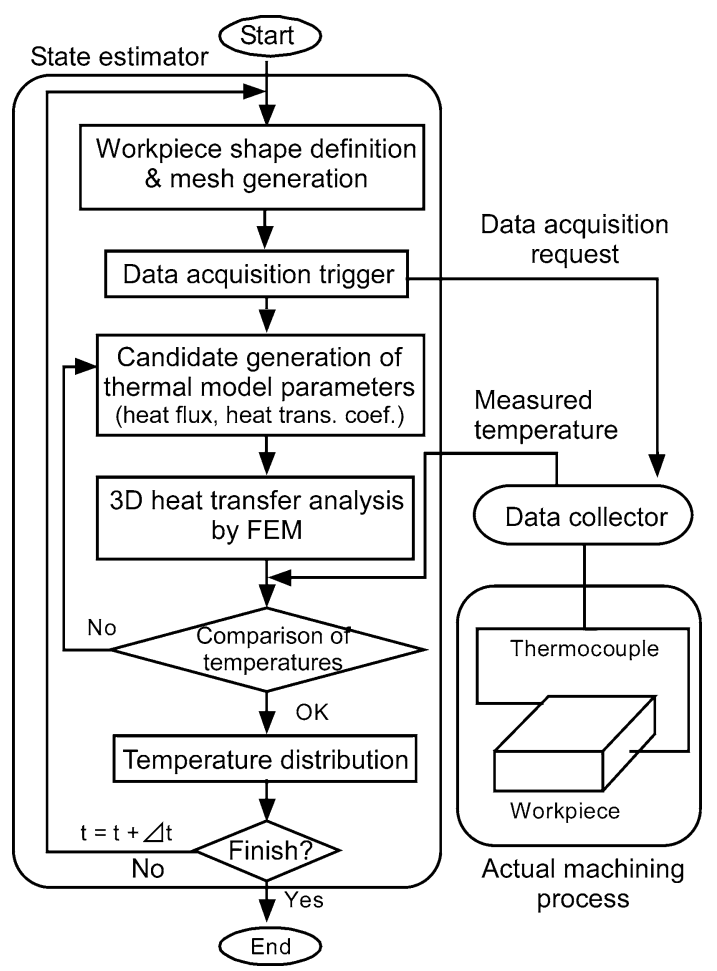

Fig. 3 Procedure of sensor-configured heat conduction simulation

to reduce the difference between the measured data and corresponding analysis results. After determining the adequate parameters, the estimation step is incremented to a next time step. Communication between state estimator and data collector is implemented based on the RS-232C protocol. All software is coded in $\mathrm{C}++$ language.

In this paper, we intend to evaluate the possibility of the proposed method. Then, only the evaluation of estimation accuracy is discussed. In order to evaluate the accuracy of estimation, it is necessary to compare several estimation trials. Then, a standard data set for estimation should be prepared. Therefore, real-time estimation is not challenged. Data collections and estimations of the machining states are separated in this evaluation. First, temperatures are measured during the machining operation without estimation. After temperature data is measured, estimation is executed by using the measured temperature data. Furthermore, the developed system cannot treat large-scale problems because the FEM analysis would require a large amount of calculation time. To reduce the calculation time of FEM analysis is a future project.

\section{Experimental Results and Discussion}

\section{1 Evaluation of temperature estimation}

In order to evaluate the proposed estimation method, the temperature distribution of the workpiece is estimated by using the developed system. Figure 4 shows an example problem for an evaluation of the adaptive estimation. The workpiece material is S50C steel. The temperature 


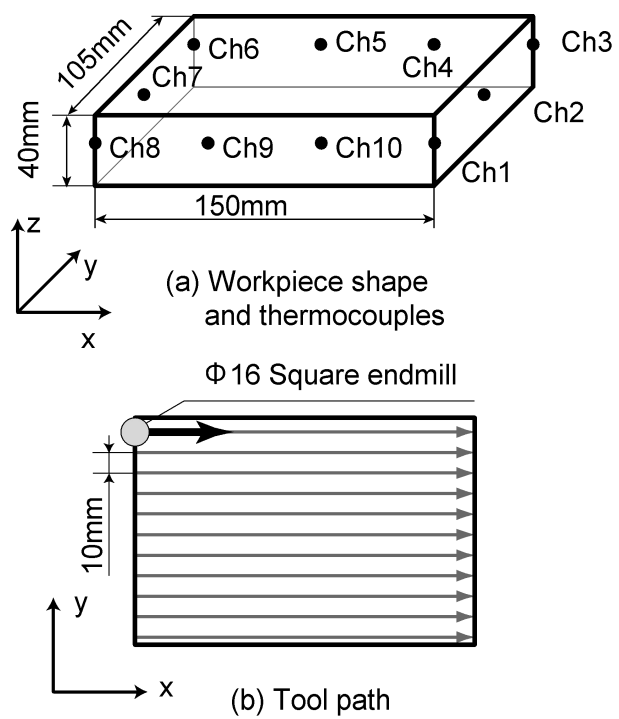

Fig. 4 Example problem

of the workpiece is measured every 5 seconds. The measurement points are illustrated in Fig. 4 (a). A single flute carbide tool, with a diameter is $16 \mathrm{~mm}$, is employed in the experiment. The tool path of the experiment is illustrated in Fig. 4(b). The temperatures of two points, (Ch6 and Ch10), are used for evaluation. The temperatures of the remaining eight points are used for state estimation. The evaluation is executed for 20 minutes.

Figure 5 shows a comparison of measured and estimated temperatures at evaluation points (Ch6 and $\mathrm{Ch} 10)$. These temperatures are not used for estimation. The simulated temperature based on the conventional FEM simulation is also illustrated in Fig. 5. In the conventional simulation, heat flux and heat transfer coefficients are determined using an example from reported research. From the experimental results, the following two points become clear:

1 ) Without sufficient preliminary parameter determination, the conventional method cannot simulate the machining process accurately. Because the differences of estimation vary by points, uniform transformation or scaling is not applicable to improving accuracy.

2 ) The proposed method can estimate the temperatures appropriately. Only the dynamically transient points show a difference. The reason can be considered to be the effect of the objective function of optimization.

Because the objective of this research is to evaluate the workpiece states, this estimation can provide sufficient accuracy of estimation. The estimated temperature fields can be visualized by using the three dimensional computer graphic technique. Figure 6 is an example of visualization.

\subsection{Influence of measurement points}

The number of temperature measurements is an important factor when utilizing this method for a realistic situation, namely, the number of measurements should be

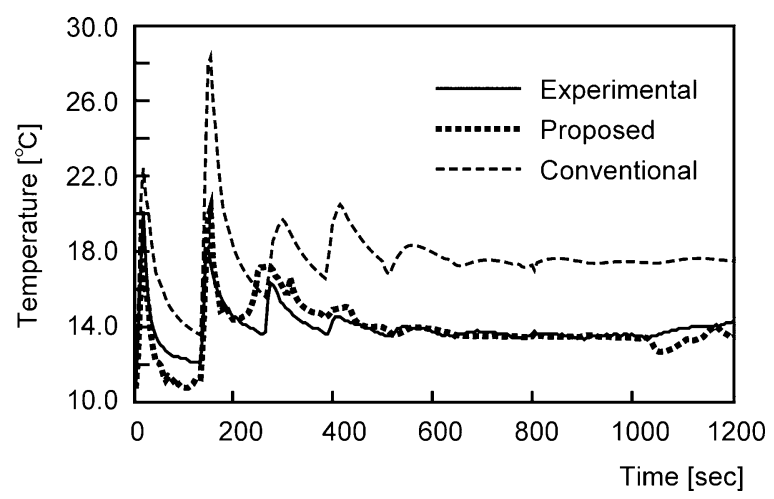

(a) Temperature at point 6

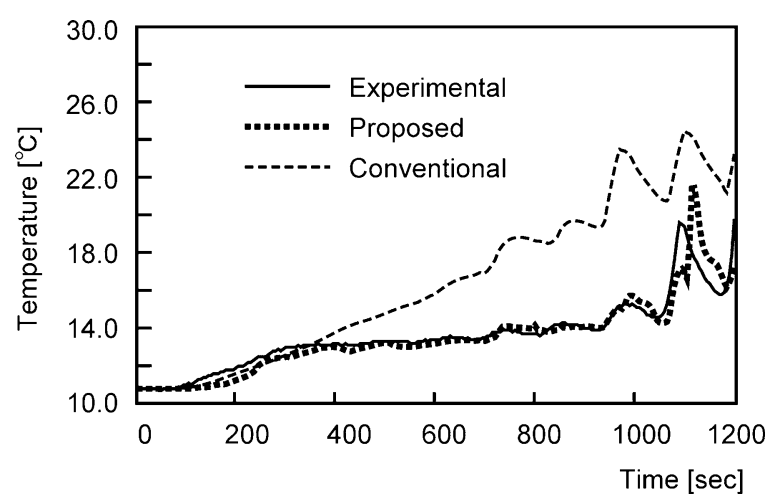

(b) Temperature at point 10

Fig. 5 Results of temperature estimation

small. The reason for this is linked to the measurement method of temperatures. The standard sensor for temperature measurement is the thermocouple. The thermocouples should be attached without interfering with either the cutting tools or fixturing equipment. Furthermore, it can be assumed that the location of the measurement points affect the estimation accuracy. Therefore, investigation was made of how the number and location of measurements influence the estimation accuracy. Figure 7 illustrates patterns of measurement points. The same experimental conditions and temperature data are used for estimation. Only evaluation points are changed to the indicated points $(\mathrm{Ch} 2$ and Ch7). Figure 8 shows the error of estimation. The error is calculated as the mean square value of difference at the evaluation points ( $\mathrm{Ch} 2$ and $\mathrm{Ch} 7)$.

The results show that too small a number of measurements results in unstable estimation. The results of patterns (i) and (ii) indicate that the location of measurement has an effect, especially in the case of a small number of measurements. In addition, in the case of small a number of measurements, it is difficult to distinguish the local measurement variation and the global temperature change. This influence trend varies according to the workpiece shape and machining tool path. The result shows the existence of the influence of sensor location and number of measurements. Theoretical evaluation of these influences is a future work of this research. Sensitivity analysis is 


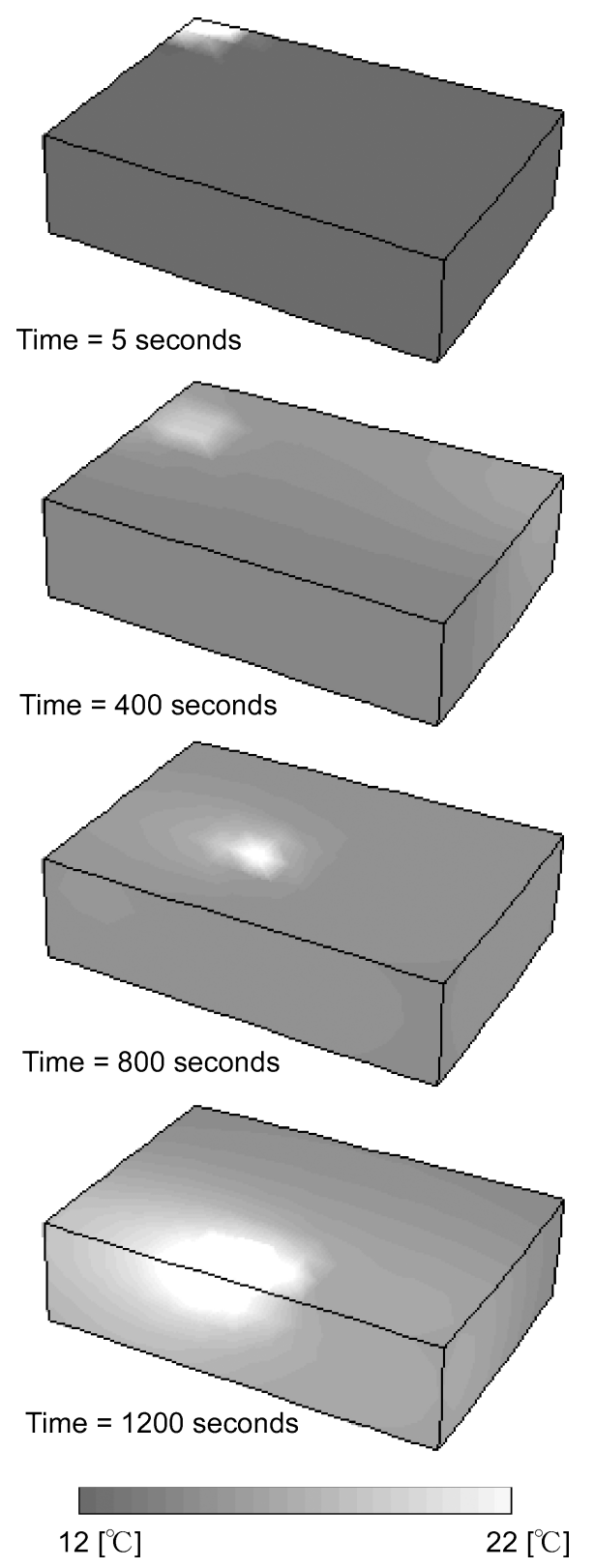

Fig. 6 Visualization of temperature distribution

expected to evaluate the influences.

\section{Conclusion}

An adaptive estimation method is proposed in order to estimate the thermal state of a workpiece in various situations. As an implementation of the proposed method, a three-dimensional transient heat conduction analysis and in-process data acquisition technique are integrated as a sensor-configured heat conductive simulation. To evaluate the proposed method, a prototype system is developed for the endmilling operation. By using the prototype system, estimation of the workpiece temperature field and the effect of measurement locations are investigated. The experimental results indicate that the developed system can estimate the thermal state accurately. It shows the possi-

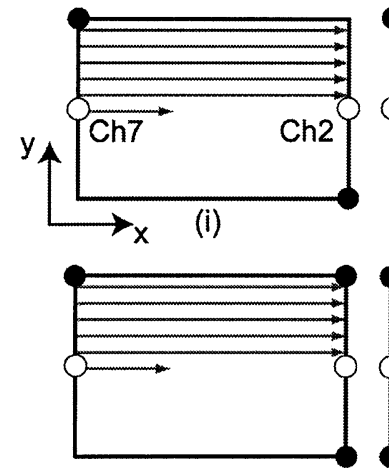

(iii)

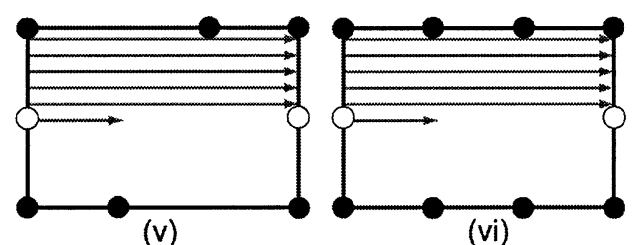

Measuring points for state estimation Measuring points for evaluation

Fig. 7 Positions of temperature measurement

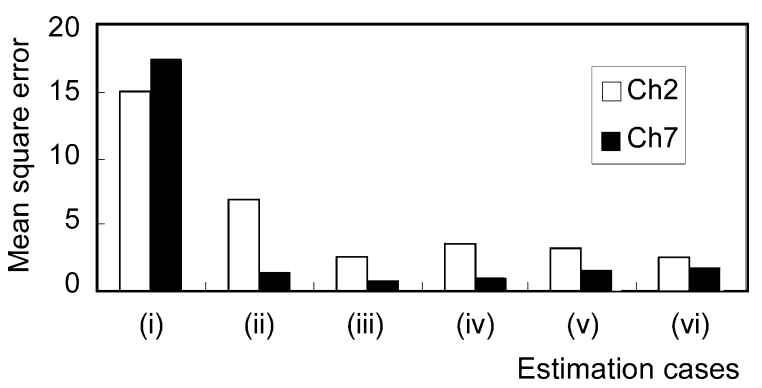

Fig. 8 Mean square error of estimated temperatures

bility of adaptive parameter estimation. The adaptability enables to reflect the situation of actual machining process to the visualized information. The systematic reduction of the number of measurement points and adaptation to large-scale problems are future works of this research.

\section{Acknowledgements}

This research was partially supported by the Ministry of Education, Science, Sports and Culture, Grant-in-Aid for Young Scientists (B), 16760092, 2004.

\section{References}

( 1 ) ISO12100, Safety of Machinery-Basic Concepts, General Principles for Design, (2003).

( 2 ) Azuma, R.T., A Survey of Augmented Reality, Teleoperators and Virtual Environment, Vol.6, No.4 (1997), pp.335-385.

( 3 ) Houten, F.J.A.M. van and Kimura, F., The Virtual Maintenance System: A Computer-Based Support Tool for Robust Design, Product Monitoring, Fault Diagnosis and Maintenance Planning, Annals of the CIRP, Vol.49, No.1 (2000), pp.91-94.

(4) Takata, S., Generation of Machining Scenario and Its 
Application to Intelligent Machining Operation, Annals of the CIRP, Vol.42, No.1 (1993), pp.531-534.

( 5 ) Teramoto, K., Kaneko, J., Onosato, M. and Takeuchi, Y., Machining State Visualization for Enhancing Operators' Situation Awareness, Proc. of the 37th International Seminar on Manufacturing Systems, (2004), pp.401-407.

( 6 ) Moriwaki, T., Emwardy and Wang, L., Study on Machining Error Due to Cutting Heat in Endmilling,
Memoirs of the Graduate School of Science and Technology, Kobe University, Vol.13-A (1995), pp.131140.

( 7 ) Matsumura, T. and Usui, E., Temperature Analysis of Tool and Workpiece in Milling Process, Proceedings of the 2000 Japan-USA Flexible Automation Conference, Vol.1 (2000), pp.515-520.

( 8 ) Kubo, S., Inverse Problem, (in Japanese), (1992), Baifu-kan. 